\title{
Physicochemical Properties of Chitosan Extracted from Pleurotus ostreatus and Improvement of its Antibacterial Activity by Gamma Radiation
}

\author{
Md. Tanvir Kabir ${ }^{1}$, Md. Shahinur Kabir ${ }^{1}$, Abdul Bathen Miah², Md Azadul Kabir Sarker², \\ Md. Kamruzzaman Pramanik ${ }^{2 *}$ \\ ${ }^{I}$ Department of Botany, Jahangirnagar University, Savar, Dhaka 1342, Bangladesh, ${ }^{2}$ Institute of Food and Radiation Biology, Atomic Energy Research Establishment, \\ Savar, Dhaka, Bangladesh
}

\begin{abstract}
This study was carried out to determine the physicochemical characteristics such as intrinsic viscosity (IV), molecular weight (MW), water binding capacity (WBC), and fat binding capacity (FBC) of chitosan extracted Pleurotus ostreatus fruit body. Antibacterial activity of the gamma irradiated chitosan was also determined. The intrinsic viscosity, MW, WBC and FBC of the produced chitosan were $769.69 \mathrm{ml} / \mathrm{g}, 1.8 \times 10^{5} \mathrm{Da}, 408 \%$ and $234 \%$, respectively. The fungal chitosan was subjected to different doses (viz., 5, 10, 20, 30 and $40 \mathrm{kGy}$ ) of radiation from ${ }^{60} \mathrm{Co}$ gamma source to observe the effect of gamma radiation on its antibacterial activity. After irradiation, antibacterial activity was evaluated and compared with that of non-irradiated chitosan. Non-irradiated chitosan showed moderate antibacterial activity against Bacillus subtilis ATCC 6633, Staphylococcus aureus ATCC 6538, Escherichia coli ATCC 35150, and Salmonella enteritidis ATCC 13076 whereas chitosan samples treated with 5, 10 and $20 \mathrm{kGy}$ gamma irradiation separately have shown enhanced antibacterial activity than that of non-irradiated chitosan against the above-mentioned bacteria. However, higher doses of gamma irradiation (30 kGy and $40 \mathrm{kGy})$ caused a gradual decline of the antibacterial activity.
\end{abstract}

Keywords: Chitosan, Pleurotus ostreatus, intrinsic viscosity, molecular weight, gamma radiation, antibacterial activity.

\section{Introduction}

Chitin is a structural component of the exoskeleton of crustaceans and insects, skeleton of sponges and inner skeleton of squid and cuttlefish, and cell wall of fungi. Chitosan is a deacetylated polymer (1-4) linked D-glucosamine and N-acetylglucosamine of chitin. Chitosan is a nontoxic, biodegradable polymer of high molecular weight (MW).

The MW of chitosan may vary depending on the sources and is generally greater than one million Daltons (Da). For example, commercially used chitosan have MW ranging from $1.0 \times 10^{5}$ $1.2 \times 10^{6} \mathrm{Da}^{2}$. The MW of chitosan can be determined by chromatography $^{3}$, light scattering ${ }^{4}$ and viscometry ${ }^{5}$. among which, viscometry is the simplest and rapid method for the determination of MW of chitosan ${ }^{5,6}$.

Different physicochemical factors (viz., temperature, $\mathrm{pH}$, concentration, molecular weight, ionic strength etc.) influence the viscosity of chitosan. The solubility, biodegradability, reactivity and adsorption of chitosan depend on the amount of protonated amino groups present in the polymeric chain. Chitosan is soluble in different acids such as acetic-, nitric-, hydrochloric, perchloric- and phosphoric acid ${ }^{7-12}$. The biological activity of chitosan depends significantly on its MW and DA. MW and degree of acetylation (DA) may affect the antimicrobial activity of chitosan independently ${ }^{13}$.
In previously published reports, it was shown that gamma irradiation; produced low MW chitosan with different surface charge and even different chemical structure which increases the water solubility of chitosan ${ }^{14}$ and enhances its inhibitory effect against microorganisms ${ }^{15}$.

Chitin and chitosan are used in biomedical, pharmaceutical, biotechnological and agriculture fields as bio-agents. Due to its unique polycationic nature, chitosan had been shown to possess antimicrobial activity against several microbes ${ }^{16,17}$. For antimicrobial property, chitosan may be used in feed and cosmetic products as alternative novel natural antimicrobial substance.

In Bangladesh, some studies were carried out on the production and characterization of chitosan from shrimp ${ }^{18,19}$, but no study on production of chitosan from locally available fungi had been done so far in Bangladesh. Therefore, this study was performed to produce chitosan from a locally available fungus, Pleurotus ostreatus (oyster mushroom) belong to basidiomycete, and to determine its physicochemical characteristic and to evaluate the effect of radiation on its antibacterial activity. Besides, ionizing radiation was also applied to the fungal chitosan to improve its antibacterial activity.

\section{Materials and Methods}

Extraction of chitin and production of chitosan:

Oyster mushroom (P. ostreatus) was collected from Savar, Dhaka, Bangladesh and chitin was extracted from the fruiting bodies of 
this fungus. Chitosan was produced from the extracted chitin by alkalization method ${ }^{20,21}$. Fruit bodies of the fungus were dried in an oven set at $60^{\circ} \mathrm{C}$ for 2 days and the dried material was grinded to make powder. The powder was treated with $1 \mathrm{~N} \mathrm{NaOH}$ solution and kept at $100^{\circ} \mathrm{C}$ for $3 \mathrm{~h}$. The alkali insoluble materials (AIM) were collected by filtering the slurry. The AIM was dried in an oven at $40^{\circ} \mathrm{C}$ for 4 days. The dried AIM was then dissolved in $2 \%$ acetic acid and was kept in a water bath set at $100^{\circ} \mathrm{C}$ for $5 \mathrm{~h}$. The sample solution was centrifuged at $6,000 \mathrm{rpm}$ for 10 minutes and the supernatant was decanted into a beaker. The solution was adjusted at $\mathrm{pH} 12.0$ by $2 \mathrm{~N} \mathrm{NaOH}$ solution for precipitation. The precipitate was collected through centrifugation at $6000 \mathrm{rpm}$ for $5 \mathrm{~min}$. The precipitate was washed 4-5 times with distilled water by using the same centrifugation condition and dried in the oven at $45^{\circ} \mathrm{C}$.

Determination of intrinsic viscosity and molecular weight $(M W)$ of chitosan:

The molecular weight of chitosan was determined by Ostwald viscometer ${ }^{6}$. In this method, the viscosity of a liquid is measured by comparing the viscosity of an unknown liquid (sample solution) with the viscosity of a known solvent $(0.3 \mathrm{M}$ acetic acid $+0.2 \mathrm{M}$ sodium acetate). The viscosity of the liquid was measured by comparing the flow times of two liquids $\left(\mathrm{t}_{\text {solvent }}=\right.$ flow time of solvent and $t_{\text {sample }}=$ flow time of sample) of equal volume using the same viscometer. On the basis of these flow times, average runtime, specific viscosity $\left(\bullet_{\text {spe }}\right)$ and reduced viscosity $\left(\bullet_{\text {red }}\right)$ were calculated. A graph was prepared using concentration and reduced viscosity of chitosan at $\mathrm{X}$ and $\mathrm{Y}$ coordinates, respectively. Then an extrapolation plot of reduced viscosity against chitosan concentration was made using trend line to find out the intrinsic viscosity [•], which is equal to Yintercept. The intrinsic viscosity of the sample was calculated using the following formula:

Average runtime $=($ Runtime $\mathrm{A}+$ Runtime $\mathrm{B}+$ Runtime $\mathrm{C}) / 3$

Specific viscosity $\left(\bullet_{\text {spe }}\right)=($ Sample runtime- Solvent runtime)/ Solvent runtime

Reduced viscosity $\left(\boldsymbol{\bullet}_{\text {red }}\right)=$ Specific viscosity/ Sample concentration

Intrinsic viscosity $=$ Y-intercept of the plot

The molecular weight was calculated by Mark-Houwink equation $^{22}$.

$[\bullet]=\mathrm{KM}^{ \pm}$where, $\{[\bullet]=$ Intrinsic viscosity, $\mathrm{M}=$ Molecular weight, $\mathrm{K}$ and $\pm=$ Constant $(\mathrm{K}=0.078$ and $\pm=0.76)\}$

Determination of water binding capacity $(W B C)$ :

WBC of chitosan was measured using the method described by $\mathrm{Knorr}^{23}$. WBC was determined by weighing a tube containing $0.5 \mathrm{~g}$ of chitosan. Ten milliliter water was added to it and mixed by a vortex mixer for $1 \mathrm{~min}$ to disperse the sample. The contents were left at ambient temperature for 30 min with intermittent shaking for $5 \mathrm{sec}$ after every $10 \mathrm{~min}$ and finally centrifuged at $3000 \mathrm{rpm}$ for $25 \mathrm{~min}$. Then the supernatant was decanted, the tube was weighed again. Bound water (g) was determined by subtracting the weight of centrifuge tube containing only chitosan from the weight of the tube containing water plus chitosan. WBC was calculated as follows:

WBC $(\%)=[$ Water bound $(\mathrm{g}) /$ Initial sample weight $(\mathrm{g})] \times 100$

Determination of fat binding capacity ( $F B C$ ): $\mathrm{FBC}$ of chitosan was measured using the method described by $\mathrm{Knorr}^{23}$. For the determination of FBC, same protocol described in WBC was followed and soybean oil was used instead of water. FBC was calculated as follows:

FBC $(\%)=[$ Fat bound $(\mathrm{g}) /$ Initial sample weight $(\mathrm{g})] \times 100$

\section{Irradiation of chitosan with gamma ray:}

Chitosan solution was irradiated with a series of radiation doses (i.e., 5, 10, 20, 30 and $40 \mathrm{kGy}$ ) at room temperature using a ${ }^{60} \mathrm{Co}$ Gamma source (Gamma beam, 650, AECL, Canada), situated at Atomic Energy Research Establishment (AERE), Savar, Dhaka, Bangladesh. Ceric-cereus dosimetry was performed to measure the absorbed dose level.

\section{Determination of antibacterial activity of chitosan:}

Agar well diffusion method was used for determination of the antimicrobial activity of chitosan 24,25 . To determine the antibacterial activity of chitosan, $0.5 \%$ chitosan solution was prepared in $1 \%$ acetic acid solution. The antibacterial activity of irradiated and non-irradiated chitosan samples were tested against Bacillus subtilis ATCC 6633, Staphylococcus aureus ATCC 6538, E. coli ATCC 35150, and Salmonella enteritidis ATCC 13076. The test organisms were separately cultured in nutrient broth at $37^{\circ} \mathrm{C}$ for 6 to 8 hours. A sterile cotton swab was dipped in the test tube containing the bacterial culture and then spread uniformly onto previously prepared Muller Hilton Agar plates. Wells of uniform diameter $(7.0 \mathrm{~mm})$ were bored in the MHA medium. Aliquots $(50 \mu \mathrm{l})$ of the gamma irradiated chitosan solution; nonirradiated chitosan solution and 1\% acetic acid (control) were dispensed in separate wells of the inoculated MHA plates. The plates were then incubated at $37^{\circ} \mathrm{C}$ for 20 hours. After incubation, the diameters of the zone of inhibition (in $\mathrm{mm}$ ) were measured.

\section{Results and Discussion}

In this study, the physicochemical characteristics and antibacterial activity of a fungal chitosan were determined. To the best of our knowledge, this is the first report on the antibacterial activity evaluation of chitosan produced from a fungus available in Bangladesh.

The MW of the produced chitosan was determined by viscosityaverage molecular weight determination process ${ }^{6}$. In this process, reduced viscosity was determined by using Ostwald viscometer and then intrinsic viscosity was determined from the graph (Fig.1). 


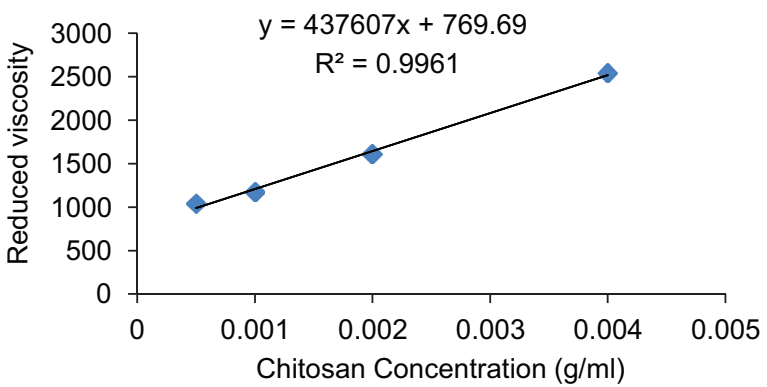

Figure 1. Determination of intrinsic viscosity of chitosan

In this case, Y-intercept of the graph suggested that the intrinsic viscosity of the fungal chitosan was $769.69 \mathrm{ml} / \mathrm{g}$. The MW was calculated by Mark-Houwink equation using this intrinsic viscosity as described previously by Wang et al. ${ }^{22}$. The MW of the chitosan from P. ostreatus was found $1.8 \times 10^{5} \mathrm{Da}$. This finding was correlated with the finding of Pochanavanich and Suntornsuk $(2002)^{26}$.

From the Table 1, it was observed that the water binding capacity of the extracted chitosan ranged from $380 \%$ to $430 \%$ (Average $408 \pm 25.80$ ). This finding did not correlate the findings of Cho, et al. (1998); they reported $458 \%$ to $805 \%$ water binding capacity of chitosan extracted from shrimp and crab shell ${ }^{27}$.

From the Table 1, it was apparent that the fat binding capacity of the extracted chitosan ranged from $196 \%$ to $262 \%$ (Average $234 \pm 33.85 \%$ ). This finding did not correlate the findings of No et al. (2000); they reported $314 \%$ to $535 \%$ fat binding capacity of chitosan ${ }^{28}$.

In a previous study it was found that chitosan has antibacterial activity against Staphylococcus aureus and Escherichia coli22 and gamma-irradiated shrimp chitosan showed elevated antibacterial activity ${ }^{19}$. Therefore, the fungal chitosan extracted from Pleurotus ostreatus was irradiated with different doses of gamma radiation to observe the effect of gamma radiation on its antibacterial activity against Bacillus subtilis ATCC 6633, Staphylococcus aureus ATCC 6538, E. coli ATCC 35150, and Salmonella enteritidis ATCC 13076.

From the Table 2, it was found that non-irradiated chitosan sample showed moderate antimicrobial activity where the zones of inhibition were 11.3, 12.0, 8.9 and $11.0 \mathrm{~mm}$, against Bacillus subtilis, Staphylococcus aureus, Escherichia coli and Salmonella enteritidis, respectively, Irradiated chitosan sample showed significant increase in activity with maximum activity 14.3, 16.0, 12.1 and $14.1 \mathrm{~mm}$, against Bacillus subtilis and Staphylococcus aureus, Escherichia coli and Salmonella enteritidi, respectively at $20 \mathrm{kGy}$ radiation applied. However, antibacterial activity of chitosan samples was decreasing gradually after $20 \mathrm{kGy}$ radiation applied.

Results from this study corroborate with the previous study where antibacterial activity of chitosan extracted from shrimp shells was increased as the irradiation dose increased up to a certain level ${ }^{29}$. A limited dose level decreased the molecular weight of chitosan in such a way that might have caused the increased membrane permeability of bacterial cells and thus inhibit their growth ${ }^{13,16}$. Therefore, dose optimization for achieving maximum antimicrobial activity is crucial and our study suggested that $20 \mathrm{kGy}$ is the optimum irradiation dose for obtaining maximum antimicrobial activity of this fungal chitosan against different Gram positive and Gram negative bacteria including food borne pathogens.

Table 1. Water binding (WB) and fat binding (FB) capacities of chitosan

\begin{tabular}{|c|c|c|c|c|c|c|c|}
\hline \multirow{2}{*}{$\begin{array}{l}\text { Expt. } \\
\text { No. }\end{array}$} & \multirow{2}{*}{$\begin{array}{c}\text { Initial } \\
\text { weight }(\mathrm{g})\end{array}$} & \multicolumn{3}{|c|}{ Water binding (WB) capacity of chitosan } & \multicolumn{3}{|c|}{ Fat binding (FB) capacity of chitosan } \\
\hline & & $\begin{array}{l}\text { Weight } \\
\text { of water } \\
\text { bound }\end{array}$ & $\begin{array}{c}\text { WBC } \\
(\%)\end{array}$ & $\begin{array}{c}\text { Ave. } \\
\text { WBC (\%) }\end{array}$ & $\begin{array}{l}\text { Weight of } \\
\text { fat bound }\end{array}$ & $\begin{array}{l}\text { WBC } \\
(\%)\end{array}$ & $\begin{array}{c}\text { Ave. } \\
\text { FBC }(\%)\end{array}$ \\
\hline 1 & 0.5 & 2.08 & 416 & & 1.48 & 196 & \\
\hline 2 & 0.5 & 2.40 & 380 & $408 \pm 25.80$ & 1.71 & 242 & $234 \pm 33.85$ \\
\hline 3 & 0.5 & 2.65 & 430 & & 1.81 & 262 & \\
\hline
\end{tabular}

Table 2. Antibacterial activity of gamma-irradiated chitosan against four common bacteria.

\begin{tabular}{lcccccc}
\hline Test organisms & \multicolumn{5}{c}{$\begin{array}{c}\text { Zone of inhibition (mm) } \\
\text { Doses of gamma-radiation (kGy) }\end{array}$} \\
\cline { 2 - 7 } & 0 & 5 & 10 & 20 & 30 & 40 \\
\hline Bacillus cereus ATCC 6633 & 11.3 & 12.8 & 13.5 & 14.3 & 12.3 & 11.8 \\
Staphylococcus aureus ATCC 6538 & 12.0 & 13.1 & 13.7 & 16.0 & 13.3 & 12.9 \\
Escherichia coli ATCC 35150 & 8.9 & 10.3 & 10.4 & 12.1 & 11.3 & 10.3 \\
Salmonella enteritidis ATCC 13076 & 11.0 & 11.9 & 12.9 & 14.1 & 12.8 & 12.0 \\
\hline
\end{tabular}




\section{Acknowledgements}

The authors would like to express heartfelt thanks to the personnel of Gamma Source Division of Atomic Energy Research Establishment (AERE), Savar, Dhaka for technical help.

\section{References}

1. Muzzarelli RAA, Rocchett R, Stanic V and Weckx M. 1997. Methods for the determination of the degree of acetylation of chitin and chitosan. Chitin handbook. pp.109-119.

2. Li Q, Dunn ET, Grandmaison EW and Goosen MF. 1992. Applications and properties of chitosan. J. Bioactive and Compatible Polym. 7 (4): 370-397.

3. Bough WA, Salter WL, Wu ACM and Perkins BE. 1978. Influence of manufacturing variables on the characteristics and effectiveness of chitosan products: Chemical composition, viscosity, and molecular weight distribution of chitosan products. Biotechnol. Bioengi. 20(12): 1931-1943.

4. Muzzarelli RAA. 1977. Some modified chitosans and their niche applications. Chitin Handbook. pp. 47-52.

5. Maghami GG and Roberts GA. 1988. Evaluation of the viscometric constants for chitosan. Macromol Chem. and Physics. 189(1): 195-200.

6. Tolaimate A, Desbrieres J, Rhazi M, Alagui A, Vincendon M and Vottero P. 2000. On the influence of deacetylation process on the physicochemical characteristics of chitosan from squid chitin. Polymers. 41(7): 2463-2469.

7. Anthonsen MW and Smidsrod O. 1995. Hydrogen ion titration of chitosans with varying degrees of $\mathrm{N}$-acetylation by monitoring induced $1 \mathrm{H}-\mathrm{NMR}$ chemical shifts. Carbohydr. Polym. 26(4): 303-305.

8. Guibal E. 2004. Interactions of metal ions with chitosan-based sorbents: a review. Separation \& Purification Technol. 38(1): 43-74.

9. Kluget AN and Denisi A. 1998. Feedback interventions: Toward the understanding of a double-edged sword. Curr. Direc. Psychol. Sci. 7(3): 67-72.

10. Kubota C, Yamakuchi H, Todoroki J, Mizoshita K, Tabara N, Barber Mand Yang X. 2000. Six cloned calves produced from adult fibroblast cells after long-term culture. Proceed. Natio. Academy Sci. 97(3): 990995.

11. K. 2006. Chitin and chitosan: functional biopolymers from marine crustaceans. Marine Biotechnol. 8(3): 203.

12. Rinaudo M. 2006. Chitin and chitosan: properties and applications. Progress Polym. Sci. 31(7): 603-632.

13. Rong HC and Horng DH. 1996. Effect of molecular weight of chitosan with the degree of deacetylation on the thermal, mechanical and permeability properties of the prepared membrane. Carbohydr. Polym. 29: $353-358$
14. Kume T and Takehisa M. 1982. Effect of gamma-irradiation on chitosan. Proceedings of the 2nd International Conference on Chitin and Chitosan. Sapporo. Japan. pp. 66-70.

15. Matsuhashi S and Kume T. 1997. Enhancement of antimicrobial activity of chitosan by irradiation. J. Sci. Food Agric. 73(2): 237-241.

16. Chen YM, Chung YC, Wang LW, Chen KT and Li SY.2002. Antibacterial properties of chitosan in waterborne pathogen. J. Env. Sci. Health. 37(7): 1379-1390.

17. Shin Y, Yoo DI and Jang J. 2001. Molecular weight effect on antimicrobial activity of chitosan treated cotton fabrics. J. Appl. Polym. Sci. 80(13): 2495-2501.

18. Hossain MS and IqbalA. 2014. Production and characterization of chitosan from shrimp waste. J. Bangladesh Agric. Univ. 12(1): 153-160.

19. Morhsed MA, Bashir AA, Khan MHK and Alam MK. 2011. Antibacterial activity of shrimp chitosan against some local food spoilage bacteria and food borne pathogens. Bangladesh J. Microbiol. 28(1): 45-47.

20. Johney J, Eagappan K and Ragunathan RR. 2017. Microbial extraction of chitin and chitosan from Pleurotus spp., its characterization and antimicrobial activity. Int. $J$. Curr. Pharm. Res. 9(1): 88-93.

21. Yang L, Li X, Lai C, Fan Y, Ouyang J and Yong Q. 2017. Fungal chitosan production using xylose rich of corn stover prehydrolysate by Rhizopus oryzae. Biotechnol. Biotechnol. Equip. 31(6): 1160-1166.

22. Wang W, Bo SQ, Li SQ and Qin W. 1991. Determination of the MarkHouwink equation for chitosans with different degrees of deacetylation. Int. J. Biol. Macromol. 13(5): 281-285.

23. Knorr D. 1982. Functional properties of chitin and chitosan. J. Food Sci. 47(2): 593-595.

24. Goy RC,Morais ST and Assis OB. 2016. Evaluation of the antimicrobial activity of chitosan and its quaternized derivative on E. coli and S. aureus growth. Revista Brasileira de Farmacognosia. 26(1): 122-127.

25. Ryan MP, Rea MC, Hill C and Ross RP. 1996. An application in cheddar cheese manufacture for a strain of Lactococcus lactis producing a novel broad-spectrum bacteriocin, lacticin 3147. Appl. Env. Microbiol. 62(2): 612-619.

26. Pochanavanich P and Suntornsuk W. 2002. Fungal chitosan production and its characterization. Letters Appl. Microbiol. 35(1): 17-21.

27. Cho YI, No HK and Meyers SP. 1998. Physicochemical characteristics and functional properties of various commercial chitin and chitosan products. J. Agric. Food Chem. 46(9): 3839-3843.

28. No H K, Lee KS and Meyers SP. 2000. Correlation between physicochemical characteristics and binding capacities of chitosan products. J. Food Sci. 65(7): 1134-1137.

29. Aktar J, Hasan MZ, Afroz T, Rashid H and Pramanik MK. 2017. Application of gamma radiation and physicochemical treatment to improve the bioactive properties of chitosan extracted from shrimp shell. Nukleonika. 62(4): 245-251. 